\title{
How far from equilibrium is active matter?
}

\author{
Étienne Fodor, ${ }^{1}$ Cesare Nardini, ${ }^{2,3}$ Michael E. Cates, ${ }^{2,3}$ Julien Tailleur, ${ }^{1}$ Paolo Visco, ${ }^{1}$ and Frédéric van Wijland ${ }^{1}$ \\ ${ }^{1}$ Université Paris Diderot, Sorbonne Paris Cité, MSC, UMR 7057 CNRS, 75205 Paris, France \\ ${ }^{2}$ SUPA, School of Physics and Astronomy, University of Edinburgh, Edinburgh EH9 3FD, United Kingdom \\ ${ }^{3}$ DAMTP, Centre for Mathematical Sciences, University of Cambridge, \\ Wilberforce Road, Cambridge CB3 OWA, United Kingdom
}

(Dated: May 30, 2016)

\begin{abstract}
Active matter systems are driven out of thermal equilibrium by a lack of generalized Stokes-Einstein relation between injection and dissipation of energy at the microscopic scale. We consider such a system of interacting particles, propelled by persistent noises, and show that, at small but finite persistence time, their dynamics still satisfy a time-reversal symmetry. To do so, we compute perturbatively their steady-state measure and show that, for short persistent times, the entropy production rate vanishes. This endows such systems with an effective Fluctuation-Dissipation theorem akin to that of thermal equilibrium systems. Last we show how interacting particle systems with viscous drags and correlated noises can be seen as in equilibrium with a visco-elastic bath but driven out of equilibrium by non-conservative forces, hence providing an energetic insight on the departure of active systems from equilibrium.
\end{abstract}

Active matter systems comprise large assemblies of individual units that dissipate energy, often stored in the environment, to produce mechanical work [1]. From the collective motion of self-propelled particles $[2,3]$ to the existence of a liquid phase in the absence of attractive forces [4-6] many intriguing phenomena have generated a continuously growing interest for active matter over the past decades [1]. Since active systems break detailed balance at the microscopic scale, they cannot be described by equilibrium statistical mechanics. However, it is often difficult to pinpoint precisely the signature of non-equilibrium physics in their emerging properties. For instance, Motility-Induced Phase Separation (MIPS), which leads to the liquid-gas coexistence of repulsive self-propelled particles, is not associated to the emergence of steady-state mass currents. A number of works have actually proposed that its large scale physics can be captured by an equilibrium theory [4, 7-9], the limits of which are heavily debated [10-12]. Even for systems where steady currents arise the connection to equilibrium physics can sometimes be maintained, as for the transition to collective motion which amounts, for simple systems, to a liquid-gas phase transition [13, 14]. More and more approaches to active matter thus partly rely on the intuition built for equilibrium systems [4, 7, 8, 12, 15-18].

Building a thermodynamic approach for active matter thus first require understanding how active systems depart from thermal equilibrium. Insight into this question was gained by studying how the Fluctuation Dissipation Theorem (FDT) breaks down in active matter [19-22]. At short time and space scales, the persistent motion of active particles typically precludes the existence of effective temperatures while at larger scales FDTs can sometimes be recovered. In living systems, the violation of FDT is used to characterize the forces generated by intracellular active processes [23-28]. The information extracted from violations of the FDT is however rather limited and non-equilibrium statistical mechanics offers more elaborate tools to quantify the departure from equilibrium. In particular, the entropy production rate quantifies the breakdown of time-reversal symmetry, whence probing the irre- versibility of the particle trajectories [29]. Hard to compute, and even harder to measure experimentally, it has been little studied in active systems [30, 31], hence the need for 'simple but not simpler' systems which offer a natural way to establish theoretical frameworks.

In this letter we study a model system of active matter which has recently attracted lots of interest [9, 32-34]. It comprises overdamped 'self-propelled' particles evolving as

$$
\dot{\mathbf{r}}_{i}=-\mu \nabla_{i} \Phi+\mathbf{v}_{i}
$$

where $i$ refers to the particle label, $\mu$ to their mobility and $\Phi$ is an interaction potential. The self-propulsion velocities $\mathbf{v}_{i}$, rather than having fixed norms as in models of Active Brownian Particles [5] (ABPs) or Run-and-Tumble Particles [35] (RTPs), are zero-mean persistent Gaussian noises of correlations $\left\langle v_{i \alpha}(t) v_{j \beta}(0)\right\rangle=\delta_{i j} \delta_{\alpha \beta} \Gamma(t)$, with greek indices corresponding to spatial components. In the simplest of cases, the $\mathbf{v}_{i}$ 's are Ornstein-Uhlenbeck processes, solutions of $\tau \dot{\mathbf{v}}_{i}=-\mathbf{v}_{i}+\sqrt{2 D} \boldsymbol{\eta}_{i}$, with $\boldsymbol{\eta}_{i}$ 's zero-mean unit-variance Gaussian white noises, so that $\Gamma(t)=D \mathrm{e}^{-|t| / \tau} / \tau$. Here $D$ controls the amplitude of the noise and $\tau$ its persistence time.

Since the temporal correlations of the noise are not matched by similar correlations for the drag, this system does not satisfy the standard generalization of the Stokes-Einstein relation to systems with memory [36]. Consequently, the system is out of thermal equilibrium and its stationary measure is not the Boltzmann weight $P_{\mathrm{B}} \equiv Z^{-1} \exp (-\beta \Phi)$. This model, to which we refer in the following as Active-Ornstein-Uhlenbeck Particles (AOUPs), shares the essential features of active systems: it correctly reproduces the behavior of passive tracers in bacterial baths $[32,37]$, leads to the standard accumulation of active particles close to confining walls [33], and shows a shifted onset of the glass transition [34]. As for many other self-propelled particles [21, 38], the limit of vanishing persistence time of AOUPs correspond to an equilibrium Brownian dynamics, since $\mathbf{v}_{i}$ reduces to a Gaussian white noise.

In the following, we characterize how the AOUPs depart from thermal equilibrium. First, we compute perturba- 
tively their steady-state at small but finite persistence time $\tau$. Surprisingly, we show that the small $\tau$ limit yields a non-Boltzmann distribution with which the particle dynamics still respects detailed-balance: The entropy production, which we compute, can indeed be shown to vanish at order $\tau$. In this regime, to which we refer as effective equilibrium, we also show that AOUPs satisfy a generalized FDT. Finally, we close this article by providing an energetic interpretation of the breakdown of detailed-balance for AOUPs.

We consider $N$ particles, propelled by Ornstein Uhlenbeck processes, interacting through a potential $\Phi$. For illustration purposes, we use pairwise repulsive forces in $2 \mathrm{~d}$

$$
\Phi=\frac{1}{2} \sum_{i, j} V\left(\mathbf{r}_{i}-\mathbf{r}_{j}\right), \quad V(\mathbf{r})=A \exp \left[-\frac{1}{1-(r / a)^{2}}\right],
$$

for which Fig. 1 shows that AOUPs exhibit Motility-Induced Phase Separation [4, 6], extending this phenomenon beyond the reported cases of RTPs [4, 22] and ABPs [5, 9, 39]. Our analytical results, however, are valid beyond this example, and hold for general potentials and dimensions. Introducing the velocities $\mathbf{p}_{i}=\dot{\mathbf{r}}_{i}$ and taking the time derivative of (1) yields

$$
\tau \dot{\mathbf{p}}_{i}=-\mathbf{p}_{i}-\left(1+\tau \mathbf{p}_{k} \cdot \nabla_{k}\right) \nabla_{i} \Phi-\sqrt{2 T} \boldsymbol{\eta}_{i},
$$

where the mobility $\mu$ is set to one. Here and in what follows, repeated indices are implicitly summed over.

We have introduced $T \equiv D / \mu$ so that taking $\tau=0$ in Eq. (3) directly maps the dynamics onto an equilibrium overdamped Langevin equation at temperature $T$. Surprisingly, there exist other equilibrium approximations of Eq. (3). First, taking $\tau=0$ in the r.h.s. maps AOUPs onto an underdamped Kramers-Langevin equation. Conversely, taking $\tau=0$ in the 1.h.s. corresponds to the Unified Colored Noise Approximation $[33,40]$ which has been shown to satisfy detailed balance [33]. Here, we propose to determine perturbatively the steady-state of AOUPs in the small $\tau$ limit, retaining both contributions of $\tau$ in Eq. (3). Rescaling time as $t=\sqrt{\tau} \tilde{t}$ and introducing the rescaled velocities $\tilde{\mathbf{p}}_{i}=\sqrt{\tau} \mathbf{p}_{i}$, the probability distribution satisfies the Fokker-Planck equation $\dot{P}\left(\left\{\mathbf{r}_{i}, \tilde{\mathbf{p}}_{i}\right\}\right)=\mathcal{L} P\left(\left\{\mathbf{r}_{i}, \tilde{\mathbf{p}}_{i}\right\}\right)$, where the operator $\mathcal{L}$ reads

$$
\begin{aligned}
\mathcal{L}=-\tilde{p}_{i \alpha} \frac{\partial}{\partial r_{i \alpha}}+\frac{1}{\sqrt{\tau}} \frac{\partial}{\partial \tilde{p}_{i \alpha}}\left[\tilde{p}_{i \alpha}+\tau \frac{\partial^{2} \Phi}{\partial r_{i \alpha} r_{j \beta}} \tilde{p}_{j \beta}\right] \\
+\frac{\partial}{\partial \tilde{p}_{i \alpha}} \frac{\partial \Phi}{\partial r_{i \alpha}}+\frac{T}{\sqrt{\tau}} \frac{\partial^{2}}{\partial \tilde{p}_{i \alpha}^{2}} .
\end{aligned}
$$

In steady-state, we propose the following ansatz for small $\tau$

$$
P \propto \exp \left[-\frac{\Phi}{T}-\frac{\tilde{\mathbf{p}}_{i}^{2}}{2 T}+\sum_{n=2}^{\infty} \tau^{n / 2} \psi_{n}\left(\left\{\mathbf{r}_{i}, \tilde{\mathbf{p}}_{i}\right\}\right)\right] .
$$

Solving for $\mathcal{L} P=0$ then leads to a consistent set of equations

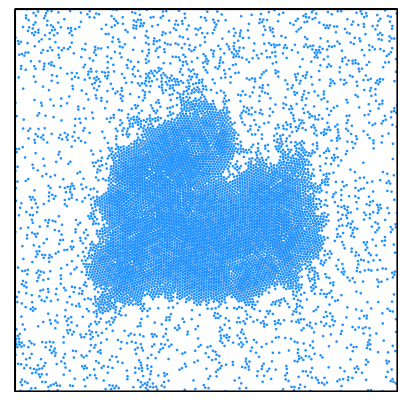

FIG. 1. AOUPs interacting via the potential (2) exhibit MIPS in a 2d box of size $L$ with periodic boundary conditions. Parameters: $A=100, a=2, N=10000, L=250, D=1000, \tau=20$

at every order in $\sqrt{\tau}$ which recursively yield

$$
\begin{aligned}
P & \propto \mathrm{e}^{-\frac{\Phi+\tilde{\mathbf{p}}_{i}^{2} / 2}{T}}\left\{1-\frac{\tau}{2 T}\left[\left(\nabla_{i} \Phi\right)^{2}+\left(\tilde{\mathbf{p}}_{i} \cdot \nabla_{i}\right)^{2} \Phi-3 T \nabla_{i}^{2} \Phi\right]\right. \\
& \left.+\frac{\tau^{3 / 2}}{6 T}\left(\tilde{\mathbf{p}}_{i} \cdot \nabla_{i}\right)^{3} \Phi-\frac{\tau^{3 / 2}}{2}\left(\tilde{\mathbf{p}}_{i} \cdot \nabla_{i}\right) \nabla_{j}^{2} \Phi+\mathcal{O}\left(\tau^{2}\right)\right\} .
\end{aligned}
$$

The distribution of positions can then be deduced by integrating (6) over velocities; this leads to a Boltzmann-like measure, $P\left(\left\{\mathbf{r}_{i}\right\}\right) \propto \exp (-\tilde{\Phi} / T)$, with an effective potential:

$$
\tilde{\Phi} \equiv \Phi+\tau\left[\left(\nabla_{i} \Phi\right)^{2} / 2-T \nabla_{i}^{2} \Phi\right]+\mathcal{O}\left(\tau^{2}\right) .
$$

In the limit of vanishing $\tau$, one recovers the standard Maxwell-Boltzmann distribution. The joint distribution of position and velocities (6) beyond this regime is our first important result. First, it shows how, for finite $\tau$, positions and velocities are correlated, in agreement with the UCNA approximation [41] but at contrast to thermal equilibrium where the energy can be separated between kinetic and potential parts. In particular, this leads to a modified equipartition theorem:

$$
\left\langle\tilde{\mathbf{p}}_{i \alpha}^{2}\right\rangle=T-\tau\left\langle\left(\nabla_{i} \Phi\right)^{2}\right\rangle_{\mathrm{B}}+\mathcal{O}\left(\tau^{2}\right),
$$

where the average $\langle\cdots\rangle_{\mathrm{B}}$ is taken with respect to the Boltzmann weigth $P_{\mathrm{B}}$. Second, the effective potential $\tilde{\Phi}$ predicts that repulsive pairwise potentials lead to effective attractive interactions, consistently with other approximation schemes $[9$, 33]. This explains why purely repulsive interactions can trigger MIPS. Note also how a pairwise potential leads to effective three-body interactions through the term $\left(\nabla_{i} \Phi\right)^{2}$. At this stage, our controlled expansion allows us to describe the static properties of AOUPs in terms of an effective Boltzmann weight (7). Interestingly, for the evolution operator (4), the asymmetry in $\tilde{\mathbf{p}_{i}}$ of the steady-state measure (6) implies that the dynamics is out-of-equilibrium [42]. This asymmetry is not captured by UCNA approximation [41] which cannot describe the non-equilibrium properties of AOUPs.

To better measure the degree of irreversibility of the dynamics, we derive its entropy production rate $\sigma$ [29]. It is obtained by comparing the probability weights associated with a given trajectory $\mathbf{r}_{i}(t)$ and its time-reversed counterpart, respectively 
denoted by $\mathcal{P}$ and $\mathcal{P}^{\mathrm{R}}$ :

$$
\sigma \equiv \lim _{t \rightarrow \infty} \frac{1}{t} \ln \frac{\mathcal{P}}{\mathcal{P}^{\mathrm{R}}}
$$

To keep the scaling in $\tau$ explicit, we work for now with the rescaled variables $\tilde{t}$ and $\tilde{\mathbf{p}}_{i}$ and use the fact that $\sigma$ is intensive in time. Using standard path-integral formalism [43], the trajectory weight can be written as $\mathcal{P}\left[\left\{\mathbf{r}_{i}(\tilde{t})\right\}\right] \sim$ $\exp \left(-\mathcal{S}\left[\left\{\mathbf{r}_{i}(\tilde{t})\right\}\right]\right)$ with

$$
\mathcal{S}=\frac{\sqrt{\tau}}{4 T} \int_{0}^{\tilde{t}} \mathrm{~d} u\left[\dot{\tilde{\mathbf{p}}}_{i}+\frac{\tilde{\mathbf{p}}_{i}}{\sqrt{\tau}}+\left(1+\sqrt{\tau} \tilde{\mathbf{p}}_{k} \cdot \nabla_{k}\right) \nabla_{i} \Phi\right]^{2} .
$$

The time-reversed trajectories are then given by $t^{\mathrm{R}}=-t$, $\mathbf{r}_{i}^{\mathrm{R}}(t) \equiv \mathbf{r}_{i}(-t)$. For $\mathbf{r}_{i}^{\mathrm{R}}(t)$ to be solution of the equation of motion (1), one then needs $\mathbf{p}_{i}^{\mathrm{R}}(t)=-\mathbf{p}_{i}(-t)$. The probability $\mathcal{P}^{\mathrm{R}}$ is then simply obtained by injecting these expressions in (10). The entropy production rate is thus given by $\sigma \sim \delta \mathcal{S} / t$ where $\delta \mathcal{S}$ is the difference between the forward and backward actions [44]. All in all, the entropy production rate reads

$\sigma=-\lim _{\tilde{t} \rightarrow \infty} \frac{\sqrt{\tau}}{T \tilde{t}} \int_{0}^{\tilde{t}} \mathrm{~d} u\left(\dot{\tilde{\mathbf{p}}}_{i} \cdot \nabla_{i}\right)\left(\tilde{\mathbf{p}}_{j} \cdot \nabla_{j}\right) \Phi=\frac{\sqrt{\tau}}{2 T}\left\langle\left(\tilde{\mathbf{p}}_{i} \cdot \nabla_{i}\right)^{3} \Phi\right\rangle$,

where the last equality follows from integrating by parts [45] and using the equality between time and ensemble averages in steady-state [46]. Interestingly, the entropy production rate exactly vanishes when $\Phi$ is quadratic in the particle displacements, hence showing that AOUPs are in this case an equilibrium model. Their steady-state is however not the Boltzmann measure $P_{\mathrm{B}}$, which explains the difficulty of defining a temperature in this case [21]. As a result, the anharmonicity of the potential acts as a control parameter for the nonequilibrium nature of AOUPs.

The entropy production rate can also be computed in the small $\tau$ limit, using the stationary distribution (6) to evaluate the correlation function appearing in Eq. (11). Going back to the initial variables, the entropy production rate is given by

$$
\sigma=\frac{T \tau^{2}}{2}\left\langle\left(\nabla_{i} \nabla_{j} \nabla_{k} \Phi\right)^{2}\right\rangle_{\text {в }}+\mathcal{O}\left(\tau^{3}\right)
$$

The first non-vanishing contribution to $\sigma$ comes from the $\tau^{3 / 2}$ correction in the steady-state measure (6). At order $\tau$, we thus have a non-Boltzmann steady-state given by the first line of (6), or equivalently by (7) in position space, with a vanishing entropy production rate. In such a regime, the AOUPs are effectively a non-thermal equilibrium model which is the central result of this letter.

Let us now discuss the practical consequences of this effective equilibrium dynamics. Oscillatory shear experiments have become an increasingly standard procedure to sample the microrheology of active systems [23, 24, 47, 48]. In this context, the violation of the equilibrium FDT has proven a natural measure of the distance to equilibrium [19, 20, 49]. Let us consider that an external operator perturbs the dynamics by applying a small constant force $\mathbf{f}_{j}$ on the particle $j$, hence modifying the potential $\Phi$ as $\Phi \rightarrow \Phi-\mathbf{f}_{i} \cdot \mathbf{r}_{i}$. We define the response function $R$ as

$$
\left.R_{i \alpha j \beta}(t, s) \equiv \frac{\delta\left\langle r_{i \alpha}(t)\right\rangle}{\delta f_{j \beta}(s)}\right|_{\mathbf{f}=0} .
$$

Following standard procedures [50], we can use the dynamic action formalism and the fact that $\delta \mathcal{P}=-\delta \mathcal{S}$.P to rewrite the response as

$$
R_{i \alpha j \beta}(t, s)=-\left\langle\left. r_{i \alpha}(t) \frac{\delta \mathcal{S}}{\delta f_{j \beta}(s)}\right|_{\mathbf{f}=0}\right\rangle .
$$

The perturbed dynamics of the AOUPs is readily given by

$$
\tau \dot{\mathbf{p}}_{i}=-\mathbf{p}_{i}-\left(1+\tau \mathbf{p}_{k} \cdot \nabla_{k}\right) \nabla_{i} \Phi+\mathbf{f}_{i}+\tau \dot{\mathbf{f}}_{i}-\sqrt{2 T} \boldsymbol{\eta}_{i}
$$

so that the dynamical action $\mathcal{S}$ becomes

$$
\mathcal{S}=\frac{1}{4 T} \int_{0}^{t} \mathrm{~d} u\left[\left(1+\tau \frac{\mathrm{d}}{\mathrm{d} u}\right)\left(\mathbf{p}_{i}+\nabla_{i} \Phi-\mathbf{f}_{i}\right)\right]^{2} .
$$

The response function is then given by

$$
\begin{aligned}
R_{i \alpha j \beta}(t, s)= & \left(1-\tau^{2} \frac{\mathrm{d}^{2}}{\mathrm{~d} t^{2}}\right)\left[-\frac{1}{T} \frac{\mathrm{d}}{\mathrm{d} t}\left\langle r_{i \alpha}(t) r_{j \beta}(s)\right\rangle\right. \\
& \left.+\frac{1}{2 T}\left(\left\langle\left. r_{i \alpha}(t) \nabla_{j \beta} \Phi\right|_{t=s}\right\rangle-\left\langle\left. r_{i \alpha}(s) \nabla_{j \beta} \Phi\right|_{t}\right\rangle\right)\right] .
\end{aligned}
$$

In the effective equilibrium regime, the vanishing entropy production tells us that the dynamics is symmetric under time reversal so that the second line of Eq. (17) vanishes and the response function finally reads:

$$
R_{i \alpha j \beta}(t, s)=-\frac{1}{T} \frac{\mathrm{d}}{\mathrm{d} t}\left\langle r_{i \alpha}(t) r_{j \beta}(s)+\tau^{2} p_{i \alpha}(t) p_{j \beta}(s)\right\rangle .
$$

We have thus derived a generalized FDT which holds in the small $\tau$ limit where the AOUPs are effectively in equilibrium, though not with respect to the Boltzmann measure $P_{\mathrm{B}}$. This explains the atypical form of the correlation function entering, which involves the position autocorrelation function, as in thermal equilibrium, along with the velocity autocorrelation function. Note that, as in equilibrium, this FDT is completely independent of the interaction potential $\Phi$, so that it should be measurable without knowledge of the intimate details of particle interactions.

To test whether a finite $\tau$ regime exists where our generalized FDT can indeed be measured, we consider a perturbation $\Phi \rightarrow \Phi-f \varepsilon_{i} x_{i}$ where $\varepsilon_{i}$ is a random variable equal to \pm 1 with equal probability [49]. We measure the susceptibility $\chi(t) \equiv \int_{0}^{t} \mathrm{~d} s R_{i x i x}(t, s) / N$ in simulations of AOUPs interacting with the repulsive potential (2). Our modified FDT predicts that

$$
N T \chi(t)=\left\langle\left[x_{i}(0)-x_{i}(t)\right] x_{i}(t)\right\rangle+\tau^{2}\left\langle\left[\dot{x}_{i}(0)-\dot{x}_{i}(t)\right] \dot{x}_{i}(t)\right\rangle,
$$

which is shown to be valid at small $\tau$ in Figure 2a. 


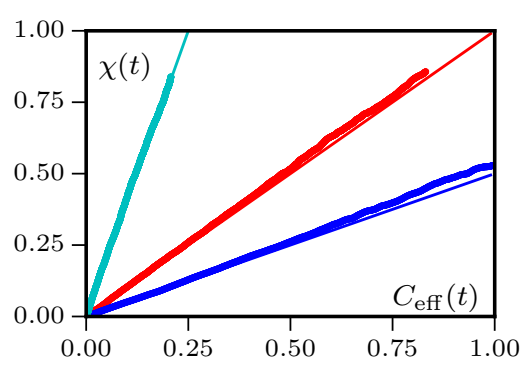

FIG. 2. Parametric plot between the susceptibility $\chi(t)$ and the correlation function $C_{\text {eff }}(t)=\left\langle x_{i}(t)\left(x_{i}(t)-x_{i}(0)\right)+\tau^{2} \dot{x}_{i}(t)\left(\dot{x}_{i}(t)-\right.\right.$ $\left.\left.\dot{x}_{i}(0)\right)\right\rangle$ for $N$ AOUPs interacting via the potential (2). The particles experience a stiff harmonic potential when they try to exit a box of linear size $L$. Parameters: $L=30, N=720, \tau=0.01, A=20$. Blue, red and cyan dots correspond to $T=2,1,0.25$ and the solid line correspond to the theoretical prediction (19).

Note that an entropy production rate $\sigma$ of order $\tau^{2}$ means that trajectories of length $\propto \tau^{-2}$ lead to an overall entropy production of order one. Since we are working in the small- $\tau$ but-finite- $D$ limit, diffusive equilibration times $\ell^{2} / D$ remain of order one, which legitimates the claim of an effective equilibrium regime. Nevertheless, we expect our FDT to break down in the long time limit. The connection between $\sigma$ and the breakdown of the FDT can be rationalized through a simple generalization of the Harada-Sasa relation [51, 52]

$$
\sigma=\frac{1}{T} \int \frac{\mathrm{d} \omega}{2 \pi} \frac{\omega}{\mu}\left[2 T R_{i \alpha i \alpha}^{\prime \prime}(\omega)+\omega C(\omega) K(\omega)\right],
$$

where $R^{\prime \prime}(\omega)$ is the imaginary part of the response in Fourier space, $K(\omega) \equiv 1+(\omega \tau)^{2}$ is the inverse of the noise correlator $\Gamma(t)$ in Fourier space and $C(t) \equiv\left\langle\mathbf{r}_{i}(t) \cdot \mathbf{r}_{i}(0)\right\rangle$. Interestingly, the measurement of $\sigma$ through (20) no longer requires the knowledge of the interaction potential $\Phi$, at variance with the use of Eq. (11).

To get more physical insight into our effective equilibrium regime and its breakdown as $\tau$ increases, let us now discuss the energetics of AOUPs. Active matter is traditionnaly regarded as a non-equilibrium medium because injection and dissipation of energy are uncorrelated. Indeed the former stems from the conversion of some form of stored energy while the latter results from the friction with the surrounding medium. Consequently, fluctuations and dissipations are not constrained by any form of Stokes-Einstein relations. For driven Langevin processes, the non-equilibrium nature of the dynamics can be measured as a mean heat transfer between particles and thermostat $[53,54]$. This leads to a standard definition of dissipation $J$ as the imbalance between the power injected by the thermal noise and the one dissipated via the drag force. This definition furthermore provides an energetic interpretation of the entropy production since $J=T \sigma$ [54]. A naive generalization of this reasoning to AOUPs would lead to the definition of dissipation through

$$
J=\mu^{-1}\left\langle\mathbf{p}_{i} \cdot\left(\mathbf{p}_{i}-\mathbf{v}_{i}\right)\right\rangle .
$$

It is however straightforward to see that $J=\left\langle\mathbf{p}_{i} \cdot \nabla_{i} \Phi\right\rangle=$ $\mathrm{d}\langle\Phi\rangle / \mathrm{d} t$ which necessarily vanishes in steady-state.

The breakdown of detailed balance for AOUPs is indeed not linked to a mean heat flux extracted from an equilibrated bath but from the apparent lack of generalized FDT between damping and fluctuations in (1). To get more insight on the entropy production rate $\sigma$, we remark that this dynamics is equivalent to

$$
K * \dot{\mathbf{r}}_{i}=\boldsymbol{\xi}_{i}-\mu K * \nabla_{i} \Phi,
$$

where $K(t)=\left[1-\tau^{2}(\mathrm{~d} / \mathrm{d} t)^{2}\right] \delta(t), *$ denotes time convolution, and we have introduced the noise term $\boldsymbol{\xi}_{i} \equiv K * \mathbf{v}_{i}$. The lhs of (22) corresponds to the damping of a visco-elastic fluid with memory kernel $K$. The first term on the rhs is a fluctuating force whose variance is:

$$
\left\langle\xi_{i \alpha}(t) \xi_{j \beta}(0)\right\rangle=\delta_{i j} \delta_{\alpha \beta} K(t),
$$

since by definition $(K * \Gamma)(t)=\delta(t)$. The damping and fluctuating forces appearing in (22) thus satisfy a generalized Stokes-Einstein relation [36]. They correspond to the connection of particles with an equilibrated visco-elastic bath, for which the standard definition of the dissipation applies:

$$
\mathcal{J}=\mu^{-1}\left\langle\mathbf{p}_{i} \cdot\left(K * \mathbf{p}_{i}-\boldsymbol{\xi}_{i}\right)\right\rangle .
$$

From there, simple algebra shows that $\mathcal{J}=T \sigma$, which yields a physical interpretation to $\sigma$ as the dissipation in an equilibrated bath for the dynamics (22).

Interestingly, this shows that the breakdown of detailed balance in AOUPs can be seen equivalently as resulting from a lack of generalized Stokes-Einstein relation between damping and fluctuations or from the fact that $K * \nabla_{i} \Phi$ is not a conservative force. In this second interpretation, the entropy production rate now has a standard energetic interpretation. The existence of an effective equilibrium regime for small $\tau$ is then due to the fact that $K * \nabla_{i} \Phi$ behaves as a conservative force $\nabla_{i} \tilde{\Phi}$ in this limit. The dynamics (22) with $K * \nabla_{i} \Phi$ replaced by $\nabla_{i} \tilde{\Phi}$ can be regarded as a dynamical equilibrium approximation of AOUPs; one indeed checks, for instance, that $\langle\tilde{\Phi}\rangle-\langle K * \Phi\rangle=\mathcal{O}\left(\tau^{2}\right)$ or that our generalized FDT corresponds to perturbing this equilibrium dynamics as $\tilde{\Phi} \rightarrow \tilde{\Phi}-\mathbf{r}_{i} \cdot\left(K * \mathbf{f}_{i}\right)$.

In this article we have thus shown that, as their persistence time increases, Active Ornstein-Uhlenbeck Particles do not immediately leave the realm of equilibrium physics. At short persistent time, they behave as an equilibrated visco-elastic medium with effective Boltzmann weight $P \propto$ $\exp (-\tilde{\Phi} / T)$ which differs from the thermal equilibrium $P_{\mathrm{B}} \propto$ $\exp (-\Phi / T)$. In this regime, the fact that repulsive forces lead to effective attractive interactions can directly be read in $\tilde{\Phi}$. Beyond this static result, the existence of an effective equilibrium regime enforces a generalized fluctuation dissipation theorem, akin to its thermal counterpart though different correlators are involved. The breakdown of this FDT for larger persistence times can be linked to a non-zero entropy production rate whose expression we have computed analytically. 
Last, we have shown how to extend the notion of dissipation to understand the breakdown of detailed balance in AOUPs.

Most of the results presented in this letter have been derived for the particular choice of noise correlator $\Gamma(t)=$ $D \mathrm{e}^{-|t| / \tau} / \tau$. Many of our results, such as Eq. (20) or the discussion on dissipation, however extends to more general correlators. Furthermore, it has recently been shown that static approximations derived for the steady-state of AOUPs capture very well the physics of ABPs [9]. It would thus be very interesting to know whether our effective equilibrium regime also extends to this system. More generally, our study suggests that when systems are driven out of thermal equilibrium by the conversion of some form of stored energy, an effective equilibrium regime may remain when the drive is moderate. This would be a first step towards a thermodynamics of Active Matter.

Acknowledgements. We thank M. Baiesi, JL Barrat, E. Bertin, O. Grossein, C. Maggi, R. di Leonardo, G. Szamel, and for interesting discussions. JT was supported by ANR project Bactterns. CN was supported by EPSRC grant Nr. $\mathrm{EP} / \mathrm{J} 007404$.

[1] M. Marchetti, J. Joanny, S. Ramaswamy, T. Liverpool, J. Prost, M. Rao, and R. A. Simha, Reviews of Modern Physics 85, 1143 (2013).

[2] T. Vicsek, A. Czirók, E. Ben-Jacob, I. Cohen, and O. Shochet, Physical review letters 75, 1226 (1995).

[3] T. Vicsek and A. Zafeiris, Physics Reports 517, 71 (2012).

[4] J. Tailleur and M. E. Cates, Phys. Rev. Lett. 100, 218103 (2008).

[5] Y. Fily and M. C. Marchetti, Phys. Rev. Lett. 108, 235702 (2012).

[6] M. E. Cates and J. Tailleur, Annu. Rev. Condens. Matter Phys. 6, 219 (2015).

[7] T. Speck, J. Bialké, A. M. Menzel, and H. Löwen, Physical Review Letters 112, 218304 (2014).

[8] S. Takatori, W. Yan, and J. Brady, Physical review letters 113, 028103 (2014).

[9] T. F. F. Farage, P. Krinninger, and J. M. Brader, Phys. Rev. E 91, 042310 (2015).

[10] R. Wittkowski, A. Tiribocchi, J. Stenhammar, R. J. Allen, D. Marenduzzo, and M. E. Cates, Nature communications 5 (2014).

[11] A. P. Solon, J. Stenhammar, R. Wittkowski, M. Kardar, Y. Kafri, M. E. Cates, and J. Tailleur, Phys. Rev. Lett. 114, 198301 (2015).

[12] J. Bialké, J. T. Siebert, H. Löwen, and T. Speck, Physical Review Letters 115, 098301 (2015).

[13] A. Solon and J. Tailleur, Physical review letters 111, 078101 (2013).

[14] A. P. Solon, H. Chaté, and J. Tailleur, Physical review letters 114, 068101 (2015).

[15] S. Mallory, A. Šarić, C. Valeriani, and A. Cacciuto, Physical Review E 89, 052303 (2014).

[16] X. Yang, M. L. Manning, and M. C. Marchetti, Soft matter 10, 6477 (2014).

[17] S. C. Takatori and J. F. Brady, Phys. Rev. E 91, 032117 (2015).

[18] F. Ginot, I. Theurkauff, D. Levis, C. Ybert, L. Bocquet,
L. Berthier, and C. Cottin-Bizonne, Physical Review X 5, 011004 (2015).

[19] D. Loi, S. Mossa, and L. F. Cugliandolo, Physical Review E 77, 051111 (2008).

[20] D. Loi, S. Mossa, and L. F. Cugliandolo, Soft Matter 7, 3726 (2011).

[21] G. Szamel, Phys. Rev. E 90, 012111 (2014).

[22] A. Solon, M. Cates, and J. Tailleur, EPJST 224, 1231 (2015).

[23] D. Mizuno, C. Tardin, C. F. Schmidt, and F. C. MacKintosh, Science 315, 370 (2007).

[24] C. Wilhelm, Phys. Rev. Lett. 101, 028101 (2008).

[25] F. Gallet, D. Arcizet, P. Bohec, and A. Richert, Soft Matter 5, 2947 (2009).

[26] E. Ben-Isaac, Y. Park, G. Popescu, F. L. Brown, N. S. Gov, and Y. Shokef, Physical review letters 106, 238103 (2011).

[27] É. Fodor, M. Guo, N. S. Gov, P. Visco, D. A. Weitz, and F. van Wijland, EPL 110, 48005 (2015).

[28] W. W. Ahmed, E. Fodor, M. Almonacid, M. Bussonnier, M.H. Verlhac, N. S. Gov, P. Visco, F. van Wijland, and T. Betz, ArXiv e-prints (2015), arXiv:1510.08299.

[29] J. L. Lebowitz and H. Spohn, J. Stat. Phys. 95, 333 (1999).

[30] C. Ganguly and D. Chaudhuri, Phys. Rev. E 88, 032102 (2013).

[31] D. Chaudhuri, Phys. Rev. E 90, 022131 (2014).

[32] N. Koumakis, C. Maggi, and R. Di Leonardo, Soft Matter 10, 5695 (2014).

[33] U. Marini Bettolo Marconi and C. Maggi, Soft Matter 11, 8768 (2015).

[34] G. Szamel, E. Flenner, and L. Berthier, Phys. Rev. E 91, 062304 (2015).

[35] M. J. Schnitzer, Physical Review E 48, 2553 (1993).

[36] R. Kubo, Reports on Progress in Physics 29, 255 (1966).

[37] C. Maggi, M. Paoluzzi, N. Pellicciotta, A. Lepore, L. Angelani, and R. Di Leonardo, Phys. Rev. Lett. 113, 238303 (2014).

[38] J. Tailleur and M. Cates, EPL (Europhysics Letters) 86, 60002 (2009).

[39] G. S. Redner, M. F. Hagan, and A. Baskaran, Physical review letters 110, 055701 (2013).

[40] P. Jung and P. Hänggi, Phys. Rev. A 35, 4464 (1987).

[41] U. M. B. Marconi, N. Gnan, M. Paoluzzi, C. Maggi, and R. Di Leonardo, Scientific Reports 6, 23297 (2016).

[42] C. W. Gardiner et al., Handbook of stochastic methods, Vol. 3 (Springer Berlin, 1985).

[43] L. Onsager and S. Machlup, Phys. Rev. 91, 1505 (1953).

[44] Note that we did not carefully specify the time-discretization of the Langevin equation (3) since it does not affect $\sigma$.

[45] Note that temporal boundary terms, appearing for instance after integration by parts, do not contribute to $\sigma$ thanks to the $1 / t$ prefactor.

[46] We assume that this standard result valid for the equilibrium Kramers operator extends to its slightly modified version (4).

[47] D. Robert, T.-H. Nguyen, F. Gallet, and C. Wilhelm, PLoS ONE 5, e10046 (2010).

[48] W. W. Ahmed, É. Fodor, and T. Betz, BBA - Molecular Cell Research 1853, 3083 (2015).

[49] D. Levis and L. Berthier, EPL 111, 60006 (2015).

[50] L. F. Cugliandolo, J. Phys. A 44, 483001 (2011).

[51] T. Harada and S.-i. Sasa, Phys. Rev. E 73, 026131 (2006).

[52] The result (20) is related to, but distinct from, a recent extension of the Harada-Sasa relation to continuous active field theories; E. Fodor et al., manuscript in preparation.

[53] K. Sekimoto and S.-i. Sasa, J. Phys. Soc. Jpn. 66, 3326 (1997).

[54] K. Sekimoto, Journal of the Physical Society of Japan 66, 1234 (1997). 